\title{
Modifications à la psychothérapie focalisée sur le transfert (PFT) pour le traitement des adolescents avec une identité diffuse
}

\section{Treatment of Adolescents with Identity Diffusion : A Modification of Transference Focused Psychotherapy Modificaciones a la psicoterapia focalizada en la transferencia (PFT) para el tratamiento de adolescentes con identidad difusa Modificações na psicoterapia focalizada na transferência (PFT) para 0 tratamento de adolescentes com uma identidade difusa}

\author{
Pamela A. Foelsch, Anna E. Odom et Otto F. Kernberg
}

Volume 33, numéro 1, printemps 2008

La psychothérapie focalisée sur le transfert : Québec-New York, $2^{\mathrm{e}}$ partie (1) et Débat : la garde partagée (2)

URI : https://id.erudit.org/iderudit/018472ar

DOI : https://doi.org/10.7202/018472ar

Aller au sommaire du numéro

Éditeur(s)

Revue Santé mentale au Québec

ISSN

0383-6320 (imprimé)

1708-3923 (numérique)

Découvrir la revue

Citer cet article

Foelsch, P. A., Odom, A. E. \& Kernberg, O. F. (2008). Modifications à la psychothérapie focalisée sur le transfert (PFT) pour le traitement des adolescents avec une identité diffuse. Santé mentale au Québec, 33(1), 37-60. https://doi.org/10.7202/018472ar

\section{Résumé de l'article}

Cet article présente une version modifiée de la psychothérapie focalisée sur le transfert (PFT) appliquée aux adolescents démontrant une identité diffuse. Cette méthode a déjà démontré son efficacité auprès d'adultes souffrant d'importants problèmes de diffusion de l'identité. Nous signalons l'importance de distinguer la crise identitaire normale à l'adolescence de la diffusion de l'identité, afin de bien repérer les adolescents qui pourraient bénéficier de ce traitement. Les principales modifications apportées à la PFT pour s'adapter aux adolescents impliquent divers changements : des changements quant à la fréquence et à la durée de certaines techniques particulières (par exemple une clarification accrue ; une plus grande attention aux relations extra-transférentielles avant d'aborder directement le transfert), des changements quant aux tactiques (par exemple l'inclusion de la famille à l'étape de l'évaluation et dans le contrat qui établit les phases de traitement, l'inclusion d'interventions de soutien dans le milieu tout en gardant la position analytique au cours des séances), des changements quant aux stratégies (par exemple tenter de lever les blocages qui nuisent au développement normal de l'intégration de l'identité, plutôt que de "forcer la maturation »). Le traitement vise à améliorer les relations de l'adolescent avec ses amis, ses parents et ses professeurs ; à clarifier ses objectifs dans la vie ; à acquérir une estime de lui-même et à être mieux préparé à s'investir dans les relations amoureuses. 


\title{
Modifications à la psychothérapie focalisée sur le transfert (PFT) pour le traitement des adolescents avec une identité diffuse
}

\author{
Pamela A. Fœlsch* \\ Anna E. Odom** \\ Otto F. Kernberg***
}

\begin{abstract}
Cet article présente une version modifiée de la psychothérapie focalisée sur le transfert (PFT) appliquée aux adolescents démontrant une identité diffuse. Cette méthode a déjà démontré son efficacité auprès d'adultes souffrant d'importants problèmes de diffusion de l'identité. Nous signalons l'importance de distinguer la crise identitaire normale à l'adolescence de la diffusion de l'identité, afin de bien repérer les adolescents qui pourraient bénéficier de ce traitement. Les principales modifications apportées à la PFT pour s'adapter aux adolescents impliquent divers changements: des changements quant à la fréquence et à la durée de certaines techniques particulières (par exemple une clarification accrue; une plus grande attention aux relations extra-transférentielles avant d'aborder directement le transfert), des changements quant aux tactiques (par exemple l'inclusion de la famille à l'étape de l'évaluation et dans le contrat qui établit les phases de traitement, l'inclusion d'interventions de soutien dans le milieu tout en gardant la position analytique au cours des séances), des changements quant aux stratégies (par exemple tenter de lever les blocages qui nuisent au développement normal de l'intégration de l'identité, plutôt que de «forcer la maturation»). Le traitement vise à améliorer les relations de l'adolescent avec ses amis, ses parents et ses professeurs ; à clarifier ses objectifs dans la vie; à acquérir une estime de lui-même et à être mieux préparé à s'investir dans les relations amoureuses.
\end{abstract}

'adolescence est une période de grande croissance physique et de changements hormonaux importants qui résultent dans la maturation génitale. Il s'agit aussi d'une période d'intégration des

* Assistant-professeure de psychologie clinique en psychiatrie, Weill Medical College of Cornell University.

** Professeure adjoint, School for Social Work, Smith College, boursière post-doctorale, Weill Medical College of Cornell University.

*** Directeur, Personality Disorders Institute, The New York Presbyterian Hospital, Westchester Division, Professeur de psychiatrie, Weill Medical College of Cornell University, responsable de la formation et analyste superviseur au Columbia University Center for Psychoanalytic Training and Research. 
identifications de la petite enfance aux expériences subséquentes et aux identifications associées, aux pulsions libidinales et agressives, aux aptitudes et aux rôles sociaux disponibles, le tout s'inscrivant dans un contexte de plus grande individuation par rapport à la famille et d'autonomisation personnelle accrue (Blos, 1967; Coleman, 1993 ; Erikson, 1950, 1982; Steinberg, 2001). Bien que la plupart des adolescents forment des relations interpersonnelles satisfaisantes et valorisantes, qu'ils réussissent bien au travail et à l'école, qu'ils établissent leurs premières relations amoureuses et qu'ils réalisent certains buts personnels, une minorité d'adolescents n'y arrive pas (Chabrol et Leichsenring, 2006; Cohen et al., 1993; Fleming et Offord, 1990; Rutter, 1986). De tels adolescents courent de grands risques de développer divers problèmes dans leur adaptation à l'école et au travail, ainsi que dans leurs relations familiales, intimes et interpersonnelles (Besser et Blatt, 2007; Borenstein, 1992; Orbach, 2007). La diffusion de l'identité et les pathologies qui lui sont concomitantes contribuent de façon significative à favoriser cette adaptation problématique.

Paulina Kernberg et ses collègues (2000) ont dégagé un modèle pour comprendre la pathologie de l'identité chez les enfants et les adolescents. Dans son approche de l'adolescence, celle-ci a particulièrement porté attention à la différenciation des adolescents aux prises avec des crises identitaires normales de ceux aux prises avec une diffusion de l'identité. Une crise identitaire se résorbe habituellement en une identité normale et consolidée avec un fonctionnement flexible et adaptatif, alors que l'on croit que la diffusion de l'identité est à la base de pathologies subséquentes de la personnalité menant à un grand éventail de comportements inadaptés et dysfonctionnels. Le traitement que l'on présente ici a été élaboré pour soigner des adolescents avec une identité diffuse, afin d'améliorer l'intégration de l'identité et ainsi favoriser un fonctionnement et des comportements adaptatifs. Ce traitement vise à améliorer les relations de l'adolescent avec ses amis, ses parents et ses professeurs; à l'aider à clarifier ses objectifs dans la vie ; à acquérir une estime de lui-même et à être mieux préparé à s'investir dans les relations amoureuses.

C'est à la lumière de psychothérapies réalisées ou en cours que cet article présente brièvement l'importance de bien cerner les perturbations de l'identité vécues à l'adolescence. Nous décrivons le rôle crucial de la distinction entre la «crise d'identité normale» et «la diffusion de l'identité», un exercice essentiel pour repérer correctement les adolescents à qui ce traitement convient. Enfin, nous décrivons les modifications particulières à apporter à la PFT — une thérapie qui a déjà fait 
preuve de son efficacité auprès d'adultes ayant une importante diffusion de l'identité (Clarkin et al., 1999; Clarkin et al., 2002; Clarkin et al., 2006; Clarkin et al., 2004; Clarkin et al., sous presse) — pour l'appliquer au traitement des adolescents.

\section{Importance}

Il y a des psychothérapies particulières pour différentes sortes de maladies psychiatriques (Burns et al., 1999; Longigan et al., 1998; Weisz et al., 2004), mais très peu d'entres elles ont été évaluées empiriquement par rapport au traitement des adolescents (Michael et Crowley, 2002; Weisz et al., 2006). Certaines études ont examiné la relation entre des symptômes précis (par exemple la dépression) chez les adolescents et le développement de pathologies subséquentes chez les adultes (Fergusson et al., 2005). La plupart des traitements destinés aux adolescents ont été développés en modifiant des traitements conçus à l'origine pour les adultes, pour cibler une symptomatologie particulière. Mufson et ses collègues (1999) ont modifié la psychothérapie interpersonnelle (PI) pour traiter la dépression chez les adolescents. On a adapté la thérapie dialectique comportementale (TDC) pour traiter les adolescents suicidaires. On a aussi élaboré une thérapie familiale complémentaire (Miller et al., 2002; Miller, 1999; Woodberry et al., 2002). Toutefois, ni la PI ni la TDC ne prétendent cibler les perturbations hypothétiques de l'identité sous-jacentes qui pourraient mener aux comportements et aux symptômes manifestes que visent ces traitements.

Paulina Kernberg (2000) a soutenu que les adolescents caractérisés par une prédominance de la symptomatologie du trouble de la personnalité limite (TPL) souffrent de plusieurs déficiences fonctionnelles retrouvées chez les adultes ayant un diagnostic de TPL. En fait, les études ont constaté que le TPL chez les adolescentes ressemble au TPL chez les adultes (Bradley et al., 2005). Becker et ses collègues (2002) ont soutenu que les perturbations de l'identité et les dérèglements affectifs (par exemple la colère incontrôlée et l'instabilité affective) chez les adolescents étaient les plus importants symptômes qui conduisent à un diagnostic précis de TPL. Plusieurs études ont constaté que la fréquence d'un TPL chez les adolescentes se situait entre $11,5 \%$ et $18 \%$ (Bernstein et al., 1993; Chabrol et al., 2001). On considère que le TPL est une source de déficience à long terme (Widiger et Weissman, 1991). Il est un syndrome prévalent, chronique et invalidant que l'on associe à une utilisation accrue des services médicaux et psychiatriques (Lenzenweger et al., 1997; Torgersen et al., 2001 ; Skodol et al., 2002), 
et à l'augmentation de la prévalence de comportements suicidaires et d'automutilation (McGlashan, 1986; Stone, 1993 ; Clarkin et al., 1983). Le TPL est un signe avant-coureur du développement de troubles de l'axe I selon le DSM (par exemple la dépression, l'anxiété) chez les jeunes adultes (Westen, 2006; Bernstein et al., 1993) et effets négatifs des traitements de ces troubles (Clarkin, 1996). Le TPL est aussi associé à une diminution de la réponse à la pharmacothérapie (Soloff, 2000). Bien que l'on ait fait des efforts pour aborder les aspects pathologiques du TPL propres aux adolescents (P. Kernberg, 1994), ainsi que les symptômes associés (Lewinsohn et al., 1998), aucun traitement pour adolescents ne cible précisément l'identité et les pathologies qui lui sont associées.

\section{La crise d'identité comparée à la diffusion d'identité}

L'identité est un principe organisateur fondamental qui permet de fonctionner de façon relativement indépendante des autres. L'identité joue un rôle dans l'estime de soi et dans la manière de se percevoir, assurant aussi la capacité de distinguer le soi de l'autre. Il aide aux échanges sociaux et permet de saisir l'effet qu'une personne a sur une autre. Enfin, l'identité apporte une prévisibilité et une continuité de fonctionnement au sein d'un individu, à travers les situations et au fil du temps (P. Kernberg et al., 2000).

On considère la crise d'identité comme étant une contradiction entre des expériences physiques et psychologiques qui changent rapidement d'une part, et le fossé qui s'élargit entre la perception de soi et les expériences de la perception de soi par les autres, d'autre part (O. Kernberg, 1978). La crise d'identité peut survenir lorsque l'adolescent doit faire simultanément face à des rapports physiques intimes, à un choix décisif de carrière, à des compétitions énergiques et se définir luimême au plan psychosocial (Erikson, 1959).

Dans la crise d'identité, la continuité de soi traverse les situations variées et le fil du temps, malgré l'expérimentation de divers rôles. La crise se résorbe peu à peu en une identité bien intégrée. Les études ont mis en évidence les composantes suivantes de l'identité : une image corporelle réaliste, un sentiment subjectif d'être soi, des attitudes et des comportements cohérents, une continuité temporelle de l'expérience de soi, la sincérité et l'authenticité, la clarté quant à son genre, une conscience intériorisée et l'ethnicité (Akhtar et Samuel, 1996). Le processus de formation de l'identité s'accélère chez les adolescents et se termine habituellement au jeune âge adulte. Le résolution de la crise d'identité permet ainsi à l'adolescent ou au jeune adulte de cultiver des 
amitiés satisfaisantes et valorisantes, d'avoir des objectifs précis dans la vie, d'interagir correctement avec ses parents et ses professeurs, d'établir des relations intimes et sexuelles et d'avoir de l'estime pour soi.

$\mathrm{Au}$ contraire, la diffusion de l'identité (un construit théorique présumé sous-jacent à la perturbation de l'identité tel qu'observé dans le TPL (Clarkin et al., 2006) est l'« absence d'intégration du concept de soi et des personnes significatives, de sorte que le clinicien ne peut dresser le portrait de la vision que le patient se fait de lui-même et des autres personnes importantes dans sa vie (P. Kernberg et al., 2000, p. 41). L'identité diffuse comporte plusieurs caractéristiques: une perte de la capacité de se définir soi-même, des carences de fonctionnement autonome, l'absence d'intégration du concept de soi et des autres personnes significatives, le manque de résolution du stade de séparation/ individuation, une instabilité de la représentation du soi et des objets, la perte de perspective, des descriptions chaotiques de soi, des descriptions contradictoires, rigides ou stéréotypés des autres, une incapacité à se définir, une pseudo soumission ou une pseudo rébellion, un surmoi non ou mal intégré, une difficulté de s'engager dans un travail, dans des relations intimes, face à des valeurs ou à des objectifs, une sur-identification au groupe ou aux rôles et un pénible sentiment d'incohérence. Bien que présente à l'école primaire, la pathologie a tendance à se manifester à l'adolescence, alors que les structures sociales externes se relâchent (P. Kernberg et al., 2000).

La diffusion de l'identité chez l'adolescent est compatible avec la conceptualisation de l'identité diffuse chez l'adulte élaborée par Otto Kernberg (O. Kernberg, 1977 ; P. Kernberg et Kœnisgberg, 1999). Avec les modifications qui conviennent en fonction du stade de développement, la PFT conçue pour les adultes ayant une identité diffuse devrait être efficace chez les adolescents aux prises avec une diffusion de l'identité. La diffusion de l'identité est l'aboutissement du défaut de consolider l'identité à chacun des stades de l'enfance et de l'adolescence (P. Kernberg et al, 2000). En distinguant bien la crise d'identité de la diffusion de l'identité au cours de l'adolescence et en choisissant bien le traitement, on devrait en arriver à favoriser une maturation plus saine et à alimenter le développement normal et continu d'une identité intégrée.

\section{Modifications à la PFT pour le traitement des adolescents}

Pour être en mesure de dispenser ce traitement à des adolescents avec une identité diffuse, les thérapeutes doivent au moins avoir déjà travaillé auprès d'adolescents et avoir déjà abordé des troubles de la 
personnalité. Ce traitement demande aussi une compréhension de base et certaines compétences en PFT telle qu'elle est appliquée aux adultes. Pour une description complète de la PFT, veuillez consulter le manuel de traitement des adultes (Clarkin et al., 2006). Les principales modifications apportées à la PFT pour traiter des adolescents concernent les stratégies, les techniques et les tactiques mises en œuvre selon les phases du traitement.

\section{Modifications des stratégies générales}

Tel que nous l'avons déjà signalé, on ne s'attend pas à ce que les adolescents aient une identité aussi pleinement intégrée que celle des adultes. Il faut s'attendre à des degrés d'intégration différents selon l'âge de l'adolescent. Il y a donc une réorientation stratégique générale par rapport à l'objectif de la pleine intégration de l'identité visée dans le traitement de l'adulte en PFT. Chez les adolescents, on vise plutôt à lever les blocages qui nuisent au développement normal et à la maturation. Cette version modifiée de la PFT s'attaque aux manifestations de la diffusion de l'identité avant que les modèles caractériels pathologiques ne s'incrustent, que la structure pathologique de la personnalité ne se consolide et que surviennent les déficiences de fonctionnement à long-terme.

\section{Modifications durant la phase de l'évaluation}

La phase d'évaluation initiale commence de la même manière que chez l'adulte, mais avec une différence essentielle: les membres de la famille sont toujours inclus. Cette modification est nécessaire étant donné que, la plupart du temps, les adolescents vivent à la maison avec leur famille, dont ils dépendent pour leurs besoins de base et qu'on ne s'attend pas à ce qu'ils soient aussi indépendants ou autosuffisants que les adultes. Non seulement la famille fournit-elle de l'information (par exemple l'historique du développement), mais sa présence permet d'évaluer la dynamique familiale et son incidence possible sur le traitement de l'adolescent. L'importance de cet ajustement devient évidente à mesure que les changements apportés aux techniques et aux tactiques sont expliqués.

La structure de la phase d'évaluation suppose une série de séances qui, ensemble, apportent l'information nécessaire à l'établissement d'un diagnostic différentiel complet et précis et à la formulation d'une recommandation de traitement. La première rencontre inclut l'adolescent et les deux parents, s'ils sont disponibles. Des séances individuelles seront organisées avec chacun des parents, ensemble et séparés selon les 
besoins, pour obtenir de l'information et évaluer les motifs de la demande de traitement pour l'adolescent. Au départ, tous sont présents lors de l'explication du processus d'évaluation, de la description des «règles de communication» et des raisons pour lesquelles le traitement est sollicité.

Il est important de clarifier la communication, étant donné la forte probabilité de contact et de participation des personnes significatives pendant le traitement. Les «règles de communication» mentionnées plus haut comprennent la politique standard en psychiatrie en matière de confidentialité, laquelle ne peut être utilisée que dans les cas où l'adolescent constitue un danger pour lui-même ou pour autrui. Des conditions additionnelles sont toutefois posées pour la communication entre le thérapeute et les membres de la famille. Le thérapeute précise les voies de communication: toutes les informations parviennent au thérapeute, mais seule l'information autorisée par l'adolescent est transmise par le thérapeute aux autres, surtout aux parents (sauf dans les cas de danger ou de sécurité indiqués plus haut).

Après cette brève présentation, les lignes de communication étant claires et le format bien formulé, on demande aux parents de quitter la pièce et l'entrevue initiale se poursuit avec l'adolescent seul. L'adolescent participe à l'entrevue d'évaluation de la personnalité, le Personality Assessment Interview (PAI ; Selzer et al., 1987 ; P. Kernberg et al., 2000). Cette entrevue part des mêmes présomptions qui sous-tendent l'entrevue servant à évaluer l'organisation structurelle de la personnalité chez les adultes, le Structural Interview élaboré par Otto Kernberg (1977), à savoir «que l'expérience d'entrevue du patient puise dans ses fantaisies et qu'elle influence son style d'interaction avec celui qui procède à l'entrevue » (P. Kernberg et al., 2000, p. 43). L'entrevue dévoile donc la capacité de l'individu à s'organiser et à composer avec la situation de réalité, mettant ainsi en évidence le fonctionnement de sa personnalité. L'entrevue évalue le fonctionnement de la personnalité dans les domaines suivants: représentation de soi et des objets, la cognition, les affects, la capacité réflexive (ou le moi observateur), ainsi que l'empathie avec l'intervieweur. Les réponses de l'adolescent au cours de l'entrevue permettent de les classer selon une échelle qui distingue l'organisation névrotique de l'organisation limite ou de l'organisation psychotique de la personnalité. La diffusion de l'identité constitue l'indice le plus significatif de l'organisation limite de la personnalité. Les adolescents reconnus avec une identité diffuse se voient offrir une PFT. 
Contrairement à ce qui se passe chez les adultes en PFT, on accorde beaucoup plus de temps à la recherche d'informations auprès de sources connexes (par exemple les professeurs, des membres de la famille, les médecins), ce qui s'avère essentiel pour poser un diagnostic différentiel chez les adolescents. Un aspect clé du diagnostic différentiel est de localiser la pathologie primaire. Les adolescents vivent au sein de systèmes familiaux. Les problèmes que l'on observe chez eux peuvent être la manifestation d'une pathologie de la famille (par exemple la pathologie individuelle d'un parent ou des parents, ou des frères et sœurs; la configuration de la communication dans la famille). Par contre, une famille peut être troublée par l'incidence de la pathologie de l'adolescent (c'est-à-dire la pathologie de l'adolescent contamine la famille). Le transfert des parents au thérapeute aide ce dernier à poser un diagnostic différentiel.

Les parents amènent leur adolescent en thérapie pour divers motifs. Il est important d'évaluer ce qui les motive, parce que leurs motifs peuvent avoir une incidence directe sur leur soutien au traitement ou leur désapprobation. De plus, le transfert des parents au thérapeute peut prendre une multitude de formes. Certains voient le thérapeute comme un «sauveur» qui «arrangera tout» pour eux et leur enfant. D'autres parents voient le thérapeute et le traitement comme un «lieu de débarras». Ces parents «déposent» leur adolescent en thérapie, s'attendant à ce que le thérapeute «arrange tout», abandonnant leur responsabilité d'aider à améliorer la situation. D'autres parents voient plutôt le thérapeute comme un «voleur» qui leur « dérobera» l'affection de leur adolescent. Dans ces situations, le thérapeute est perçu comme une menace dans la relation avec leur enfant. Au cours de la phase d'évaluation, il est important d'observer les prédispositions dominantes des parents face au transfert, gardant à l'esprit qu'au cours du traitement, le type de transfert dominant pourrait changer à mesure que la thérapie évolue. Ces prédispositions sont repérées et servent à prédire les passages à l'acte. Ces domaines sont circonscrits par l'inclusion des parents dans le cadre du contrat, ce qui constitue une autre modification particulière à la PFT pour adulte.

Un autre aspect est de savoir qui détient le pouvoir dans la famille? Au sein des familles normales, le pouvoir a tendance à se situer chez les deux parents qui travaillent en équipe de façon coopérative et dans le respect mutuel. Les parents interpellent correctement les enfants, établissent les normes familiales et se soutiennent l'un l'autre dans les mesures disciplinaires. Les enfants en viennent à connaître une structure prévisible au sein de la famille et à la tenir pour acquis. Lorsqu'il y a un 
conflit entre les parents, la prévisibilité, le respect et le soutien mutuels peuvent être absents, ce qui peut mener au fléchissement des alliances, favorisant des situations où les enfants exercent un pouvoir accru au sein de la famille. L'enfant peut exclure l'un des parents, ou les deux, en établissant des alliances unilatérales. Dans certains cas, l'adolescent s'allie avec les autres enfants contre les deux parents. Ces mésalliances se produisent souvent quand les parents, à cause de leurs conflits, ne peuvent faire équipe pour exercer efficacement leur rôle parental. Il est important de cerner les alliances clés au sein de la famille, non seulement pour comprendre le rôle de l'adolescent dans la famille, mais aussi pour prédire le surgissement de conflits quand ces alliances sont confrontées au cours du traitement.

La plupart des familles ont des normes qui régissent les interactions entre leurs membres et le monde à l'extérieur du foyer. Bien qu'habituellement tacites, ces «règles familiales» sont puissantes. On les observe rapidement lorsque la famille interagit avec le thérapeute au cours de l'évaluation; elles dictent en quelque sorte le type de renseignements dont on fait part, qui en fait part et à quel moment. Il y a aussi les «règles de la maison» qui dictent les normes admissibles de comportement. Ces dernières régissent des domaines comme le niveau de responsabilité individuelle au sein de la maisonnée (par exemple les tâches domestiques), et quels comportements doivent faire l'objet de mesures disciplinaires. En se renseignant au sujet des «règles de la maison», le thérapeute en arrive à comprendre à quoi la famille s'attend en matière de comportement et à constater dans quelle mesure la communication de ces règles est ou non efficace, et dans quelle mesure également leur mise en application auprès des adolescents est opérante.

D'une manière ou d'une autre, les problèmes de communication sont souvent à la source des difficultés des adolescents, et c'est particulièrement le cas pour ce qui est des relations familiales. Reconnaissons cependant que cacher des aspects de son propre vécu pendant de courtes périodes aux parents fait partie du processus normal d'individuation /séparation. La fréquence et l'ampleur de cette retenue de soi indiquent toutefois le risque de pathologie. Dans les pathologies plus graves, les «secrets» de famille peuvent survenir entre les parents ou entre les parents et l'adolescent. Ils indiquent habituellement soit un certain désaccord entre les parents ou un manque de confiance fondamentale envers l'adolescent ou une pathologie importante chez celui-ci. L'objectif est d'évaluer la fonction que remplit le «secret» pour l'individu et pour la famille. 
Le statu quo de ces «secrets», de ces patterns de communication, de ces normes, de ces alliances et de ce lieu de pouvoir est susceptible d'être ébranlé par le processus d'évaluation lui-même (et de continuer à être remis en question tout au long du traitement). Il importe que le thérapeute reconnaisse ce risque et qu'il observe la réaction de chacun des individus. Ceci devient un indicateur de pronostic. Plus chacune des personnes, et la famille dans son ensemble, est en mesure de tolérer ces menaces au statu quo, meilleur sera le pronostic du traitement.

Une fois la période de collecte d'information et de diagnostic élaboré, on prévoit une rencontre avec l'adolescent et les parents pour présenter les conclusions et recommandations des examens. S'il y a diffusion de l'identité dans le contexte de comportements inadaptés ou de déficiences fonctionnelles ou symptomatiques, il est recommandé d'offrir la PFT. Pour ce qui est du diagnostic différentiel et des indications de psychothérapie, il importe de se rappeler que les adolescents sont en processus de développement, et que la présence de plusieurs traits inadaptés est donc suffisant pour justifier une intervention et un traitement, même si tous les critères du trouble ne sont pas remplis. Ceci est compatible avec l'objectif de la psychothérapie qui veut lever les blocages nuisibles au développement normal de l'identité.

\section{Introduction d'une phase avant le contrat de traitement}

Une fois la phase d'évaluation terminée et si la PFT a été recommandée, la phase de négociation du contrat préalable au traitement commence avec l'adolescent et sa famille. Il s'agit d'une phase additionnelle, nécessaire à cause de l'interdépendance entre l'adolescent et sa famille. Pour faciliter cette phase, le thérapeute doit, en l'absence de toute transgression flagrante (par exemple la présence d'agression sexuelle ou physique), garder face à la famille un préjugé favorable à l'effet que la famille a fait son possible, bien qu'elle n'utilise peut-être pas les meilleures stratégies.

Contrairement aux adultes qui ont déjà vu plusieurs thérapeutes et subi divers traitements avant d'être recommandés en PFT, les adolescents et leur famille n'ont peut-être que très peu d'expérience ou de connaissances, voire aucune, de ce que demande une psychothérapie intensive en consultation externe. Cette phase a pour objectif d'éduquer tant l'adolescent que sa famille au sujet de la nature de leurs difficultés et des attentes du traitement. On veut ainsi optimiser le potentiel de la famille pour soutenir le traitement, améliorer les résultats du traitement et atténuer le risque d'abandon en réduisant la probabilité de clivage et de «passage à l'acte» associés. 
Au cours de cette phase éducationnelle, on informe la famille et l'adolescent, de la possibilité que les interactions au sein de la famille puissent changer de manière remarquable parce que l'adolescent suit un traitement. Il n'est pas inhabituel que l'adolescent remplisse une fonction (par exemple favoriser une alliance particulière ou un lieu de pouvoir) au sein de la famille dans son rôle de «patient identifié ». Quand l'adolescent commence à changer ou à remettre en question la famille, le statu quo peut être menacé et ainsi créer un déséquilibre. Dans cette situation, il n'est pas inhabituel que les parents ou l'adolescent interrompent brusquement le traitement. On doit informer la famille de cette possibilité de réaction thérapeutique négative ou d'échec du traitement.

La famille aura peut-être à rencontrer périodiquement le thérapeute pour aborder ces difficultés. Bien qu'il ne s'agisse pas d'une thérapie familiale et que l'on ne fasse pas d'interprétation, il est important de communiquer régulièrement avec les parents pour soutenir le travail individuel de l'adolescent. Une thérapie familiale additionnelle ou une thérapie individuelle pour les parents, en réaction à leurs propres difficultés qui ont une incidence directe sur la famille ou sur l'adolescent, peuvent être concomitamment indiquées, selon le niveau de pathologie des interactions et de l'incidence négative sur l'adolescent. Les familles sont informées de la possibilité qu'une thérapie familiale ou individuelle pour les parents puisse être recommandée au cours du cheminement du traitement.

\section{Modifications à la phase du contrat de traitement}

Le contrat de traitement avec les adolescents suit le même cadre fondamental que dans le cas de la PFT chez les adultes, sauf deux modifications : l'inclusion des parents et des interventions directes dans le milieu. Il y a des responsabilités pour l'adolescent, pour les parents et pour le thérapeute. On balise «les menaces à la poursuite du traitement » en fonction des difficultés propres à la symptomatologie actuelle et à l'histoire particulière de l'adolescent. On établit un contrat de traitement pour fournir un cadre clair au sein duquel on pourra observer les écarts, les clarifier, les confronter et, en fin de compte, les interpréter.

Bien que certains aspects du contrat restent confidentiels entre le thérapeute et l'adolescent, on inclut la famille pour préciser les capacités de l'adolescent d'assumer personnellement la responsabilité de respecter le contrat de traitement. Citons l'exemple de l'assiduité aux séances de thérapie. Les adolescents vivent habituellement à la maison et on ne s'attend pas à ce qu'ils aient le même niveau d'autonomie et 
d'indépendance que les adultes. Ce manque d'autonomie a une incidence sur le degré de responsabilité personnelle que le thérapeute peut attendre de l'adolescent. Alors que l'on peut tenir pour acquis que les adultes sont entièrement responsables pour ce qui est d'arriver aux séances à l'heure, les adolescents dépendent souvent de parents ou d'autres adultes pour leur transport aux séances. Ceci illustre une manière dont les parents sont engagés dans le contrat de traitement et de son incidence sur le traitement (par exemple la manière d'interpréter le «retard» aux séances). On discute des difficultés qui peuvent trouver leur origine chez les parents (par exemple la prédisposition au transfert; les motifs du traitement) et qui pourraient nuire aux capacités de l'adolescent de respecter le cadre et le travail thérapeutique du traitement; on clarifie les attentes et on établit des limites.

Bien que nous tenions pour acquis que les parents exercent un contrôle approprié sur les adolescents, l'évaluation révèle parfois que l'adolescent a des comportements qui contrôlent les parents (par exemple des menaces de s'automutiler s'il n'obtient pas ce qu'il veut). Le contrat peut clarifier cette situation et enlever le gain secondaire du symptôme en atténuant le pouvoir qu'il exerce sur le comportement des parents. Dans les cas les plus graves, où les tentatives de suicide sont une manière de vivre caractériellement fondées sans faire partie d'un épisode dépressif important, on met alors en évidence le risque que l'adolescent puisse réussir à se suicider. L'acceptation de ce risque par les parents réduit le contrôle que l'adolescent peut exercer en menaçant de se suicider.

Une autre modification apportée à la PFT est l'inclusion dans le contrat de traitement d'interventions directes dans le milieu. Ces interventions peuvent prendre diverses formes, y compris la mise en œuvre de «règles de la maison» (qui ont été explorées au cours de la phase d'évaluation). Elles visent l'atténuation des comportements destructeurs et le soutien des normes adaptatives de la maison (par exemple couvre-feu; restriction des contacts avec certains pairs).

Certaines interventions dans le milieu servent à empêcher que les comportements autodestructeurs de l'adolescent menacent de mettre fin au traitement. Citons comme exemple l'établissement d'une entente avec un médecin pour qu'il évalue la gravité des actions autodestructrices. Il faudra peut-être aussi que le thérapeute communique avec les professeurs de l'école fréquentée afin d'établir divers systèmes de suivi ou de communication, autant entre lui et l'école et qu'entre l'école et la famille. Parfois, les thérapeutes recommandent que l'adolescent subisse des examens pour vérifier s'il éprouve des difficultés 
d'apprentissage, qu'il travaille avec un tuteur, qu'il participe à des groupes pour cultiver des habiletés sociales ou qu'il prenne part à des groupes tels qu'Alcooliques Anonymes ou Al-Anon. Ceci est compatible avec ce qui se fait en PFT pour adultes; toutefois, la fréquence des communications entre le thérapeute et ces soutiens complémentaires et leur intensité sont habituellement plus grandes dans le cas des adolescents.

Une autre forme d'intervention directe dans le milieu comprend la consolidation des forces de la famille, afin de réduire les aspects négatifs de la dynamique familiale. En s'appuyant sur les aspects positifs des normes de la famille, on peut discuter des «règles de la maison» et les modifier conjointement pour améliorer le fonctionnement familial et soutenir le travail thérapeutique de l'adolescent vers un fonctionnement amélioré. La discussion sert à clarifier les «règles de la maison» et à établir un terrain d'entente pour la famille, l'adolescent et le thérapeute. À l'occasion, la gravité des comportements ou de la pathologie exige qu'on les incorpore officiellement dans le contrat, afin de conserver le cadre de traitement global.

On clarifie la logique de toute intervention directe dans le milieu et on explore les réactions tant de l'adolescent que des parents face à ces interventions, et les difficultés qui pourraient en découler. Il y a place pour une certaine négociation, mais pour que le traitement se poursuive, il faut que les parents soutiennent et appliquent ces règles et que l'adolescent accepte de les respecter. Tous doivent accepter ces règles dans l'intérêt de l'objectif convenu, soit l'amélioration du fonctionnement de l'adolescent.

En somme, le contrat de traitement avec les adolescents est l'aboutissement d'une phase d'évaluation qui comprend la participation de la famille, et d'une phase préalable au contrat de traitement qui éduque la famille au sujet des attentes en vue du traitement, en mettant l'accent sur l'explication des obstacles possibles et l'instauration de limites qui conviennent à l'atteinte des résultats. Le contrat de traitement comme tel est une combinaison du contrat individuel tel que développé en PFT, et de la participation active des parents dans les domaines où ils partagent des responsabilités avec l'adolescent pour le maintien de la structure du traitement.

\section{Modifications à la phase traitement}

En PFT, l'approche thérapeutique se présente avec trois catégories : les stratégies, qui sont les objectifs à long terme du traitement ; les tactiques, qui permettent le maintien des conditions nécessaires au 
travail thérapeutique dans chacune des séances et les techniques, qui sont les instruments propres au traitement (Clarkin et al., 2006). Voici une brève description de ces catégories dans le contexte des modifications apportées pour traiter les adolescents.

En PFT, la principale stratégie consiste à repérer à quel moment dans le traitement sont activées les dyades clivées intériorisées de relations objectales idéalisées et persécutrices, et à les interpréter. Cette activation des dyades se produit surtout au sein du transfert où elles sont clarifiées, confrontées et éventuellement interprétées. Les dyades se caractérisent par une représentation du soi et une représentation de l'autre, liées par un affect. On reconnaît les dyades par l'affect ressenti par le patient, et par le contre-transfert du thérapeute (habituellement par le biais du mécanisme d'identification projective). Les représentations du soi et de l'autre oscillent et se défendent l'une contre l'autre, tout en restant inconscientes chez le patient. Le thérapeute doit suivre l'activation, les oscillations et l'alternance des dyades tout en essayant de discerner ce contre quoi on se bat dans le processus.

Chez les adolescents, une complication s'ajoute à cette stratégie de suivre les dyades relationnelles objectales affectivement dominantes dans le transfert. Quand les adolescents vivent à la maison avec leurs parents, l'activation et la «mise en scène » des relations d'objet intériorisées qui surviennent ont tendance à impliquer les parents dans le traitement. Ainsi, le rôle fonctionnel des parents implique habituellement des mécanismes de clivage, au sein desquels divers membres de la famille répercutent des dyades de relation d'objet projetées dans leurs liens avec le thérapeute ou avec le traitement.

Cliniquement, on l'observe surtout pour la présence aux séances. Par exemple, il y a «malentendu » entre l'adolescent et les parents quant à l'heure du rendez-vous, ou à propos de quel parent devait le conduire à la séance. Si ces transferts ne sont pas surveillés, éclaircis et confrontés, ils peuvent perturber le traitement. S'ils demeurent non résolus, les parents, agissant sur un aspect projeté de l'adolescent, peuvent servir de véhicule pour mettre brusquement fin au traitement. La stratégie est donc modifiée pour y inclure le rôle des parents dans le transfert.

Ce sont les expressions spontanées de l'adolescent qui peuvent servir au thérapeute pour surveiller l'activation des représentations clivées du soi et de l'autre, représentations idéalisées et persécutrices, activées dans la relation extra-transférentielle et dans le transfert. On s'éloigne de la PFT pour adultes dans ce contexte. On consacre plus de temps au monitoring des manifestations du monde objectal intériorisé 
dans le transfert, s'abstenant d'une analyse immédiate. De plus, on commence à faire des interprétations dans le contexte des relations extra-transférentielles en jeu, avant de les aborder directement dans le transfert lui-même.

Un exemple de la manière dont le langage de l'adolescent sert à explorer le transfert concerne une réponse typique de l'adolescent, suite aux efforts du thérapeute pour lui faire prendre conscience de sa frustration ou de sa colère au cours d'une séance. L'adolescent répond par la désobligeante monosyllabe «Ouais!». Habituellement, le thérapeute en PFT réagirait en tentant de relever et d'élucider la dépréciation implicite du thérapeute véhiculée par ce commentaire sur ce ton. Travaillant avec des adolescents, le thérapeute comprend cette dynamique, mais, dans un premier temps, il l'explore au sein de la relation extra-transférentielle en se servant de la technique de clarification avant de l'aborder directement au sein du transfert.

Le thérapeute peut demander à l'adolescent à quels moments il utilise cette expression «Ouais» avec ses pairs. Il clarifie ensuite comment les pairs réagissent et vérifie s'ils ressentent son attitude comme méprisante. Le thérapeute cherche aussi à savoir si l'adolescent est conscient de son attitude méprisante ou de sa fonction défensive. Ainsi, on rend l'adolescent conscient du mode d'interaction et de la dynamique sous-jacente en se servant de la relation extra-transférentielle comme véhicule. La clarification est la principale technique utilisée auprès des adolescents. Elle permet à l'adolescent d'accéder entièrement à la signification intériorisée et inconsciente de ses actions et l'incite à réfléchir sur lui-même. Éventuellement, cela mène à une interprétation directe au sein du transfert avec le thérapeute.

On augmente la prise de conscience de ces modèles répétitifs, conflictuels ou incohérents en recourant avec tact aux «boucles de confrontation». Cette technique approfondit certains éléments de clarification et prend la forme d'une confrontation, en commençant par demander à l'adolescent d'observer et ensuite d'expliquer son comportement, ses pensées, ses sentiments. Ce processus élucide les zones de contradictions ou d'incohérences et en fait prendre conscience à l'adolescent. Cette technique a d'abord été présentée à l'adolescent lors de l'entrevue Personality Assessment Interview pour évaluer le fonctionnement de sa personnalité, et ses capacités d'organisation et de composer avec la réalité. On s'en sert au cours du traitement pour élucider des aspects particuliers de l'ici et maintenant du vécu de l'adolescent, tout en l'invitant à réfléchir à son expérience. Les contradictions et les incohérences, les fonctions défensives et les motifs inconscients sont 
rendus conscients et servent à confronter les distorsions que l'adolescent applique à la réalité, ce qui prépare le terrain à l'interprétation.

Une fois que l'adolescent a admis et compris les modèles répétitifs qu'il applique, le thérapeute commence à lier les dyades clivées et les affects qui leur sont associés. Lorsque des dyades sont activées dans l'ici et maintenant, le thérapeute travaille à expliciter leur sens, à confronter leur nature incohérente ou contradictoire et les interprète en présentant à l'adolescent le sens hypothétique qu'il leur donne. Les premiers liens se font entre ce que l'adolescent en est venu à comprendre et son interaction avec ses pairs et avec sa famille.

Dans la phase mitoyenne du traitement, à mesure que l'adolescent tolère de mieux en mieux ces interprétations, le thérapeute commence à travailler au sein du transfert. Dans cette phase, lorsque des dyades sont activées dans l'ici et maintenant avec le thérapeute, elles sont accessibles pour identification directe et leur interprétation au sein du transfert. À mesure que l'adolescent arrive à tolérer la plus grande intensité de la relation transférentielle, la proportion de clarifications, de confrontations et d'interprétation de transfert commence à ressembler à celles en PFT chez les adultes.

Tout au long du traitement, en plus du travail en cours de séance, le thérapeute veille au fonctionnement de l'adolescent. On lève les blocages au développement normal de l'identité à l'aide des interprétations extra-transférentielles et transférentielles dans le processus thérapeutique, conjointement avec le soutien dans le milieu et le cadre de traitement qui ont été établis et maintenus. Avec le temps, l'activation du clivage et des représentations dissociées du soi et de l'autre sont contenues au sein de la relation transférentielle. Au cours de cette période, le fonctionnement externe de l'adolescent s'améliore habituellement. À mesure que sont levés les blocages à l'intégration de l'identité, le fonctionnement se normalise et les relations de l'adolescent avec ses pairs, sa famille et l'école s'améliorent. À mesure que se dissipent les problèmes du début, la phase de la fin du traitement s'amorce.

$\mathrm{Au}$ cours de cette phase de fin du traitement, on explore les expériences de l'adolescent face aux améliorations de son fonctionnement. Si l'adolescent a pris conscience des changements qu'il a effectués et qu'il est confiant que ces améliorations peuvent être maintenues, on consulte les parents et les autres personnes engagées dans le traitement (par exemple l'école). Il importe que le thérapeute explore et confirme toutes les zones de comportements autodestructeurs présents au départ et au cours du traitement, pour s'assurer que l'adolescent a 
vraiment connu une amélioration. Le thérapeute doit être convaincu que les améliorations se maintiendront dans le temps. Bien que la consultation du milieu extérieur soit un processus continu tout au long du traitement, elle est particulièrement nécessaire pendant la phase de fin du traitement. Si l'on s'entend sur le constat d'amélioration du fonctionnement de l'adolescent et que toutes les sources sont confiantes dans les gains réalisés et leur maintien, on planifie la fin du traitement et on en explore la signification avec l'adolescent.

Au cours de la phase de fin de traitement, le thérapeute transmet le message que, bien que les blocages qui nuisaient au fonctionnement immédiat de l'adolescent et à la formation de l'identité soient atténués, il se peut que d'autres blocages se présentent à l'avenir et justifient un retour en thérapie. Lors de la dernière séance avec les parents, on discute de la nécessité d'une psychothérapie ultérieure dans le contexte du développement normal. Les adolescents continuent de grandir et d'interagir avec un milieu qui continue aussi de changer à mesure que l'adolescent acquiert plus d'habiletés, davantage de responsabilités et une plus grande autonomie. L'identité de l'adolescent continue d'évoluer vers une résolution normale de la crise d'identité, et vers la formation d'une identité de soi intégrée et d'une vision intégrée des autres personnes significatives.

\section{Pistes d'avenir}

Nous effectuons actuellement un essai clinique randomisé, afin d'évaluer l'efficacité de cette approche thérapeutique pour le traitement de la diffusion de l'identité chez les adolescents, en la comparant au traitement habituel à notre clinique externe. Cette étude évalue l'efficacité de ce traitement modifié au sein d'une population de jeunes femmes âgées de 15 à 21 ans. Les sujets de notre étude affichent des comportements associés à l'identité diffuse. La mesure des résultats est axée sur les symptômes (par exemple, la dépression, la colère, l'anxiété) et le fonctionnement (par exemple, le fonctionnement interpersonnel avec les pairs, les adultes, la famille), ainsi que sur les changements de l'identité.

Étant donné la «banalisation» des comportements pathologiques par le recours à cette nouvelle caractérisation d'adolescents dits «émo », il est urgent que notre société aborde les questions associées à l'identité. Les cliniciens qui savent distinguer la crise d'identité de la diffusion de l'identité peuvent repérer les adolescents qui ont besoin de ce traitement spécialisé, et ainsi aider à augmenter les chances que l'adolescent se libère des blocages qui freinent sa croissance normale et son 
développement optimal. Notre recherche évalue l'efficacité de la PFT qui cible l'identité pour améliorer le fonctionnement. Nous espérons ainsi fournir une option thérapeutique, alors qu'elles sont plutôt rares, et, de toute urgence, nécessaires.

\section{Références}

AKhtar, S., Samuel, S., 1996, The concept of identity developmental origins, phenomenology, clinical relevance and measurement, Harvard Review of Psychiatry, 3, 5, 254-267.

American Psychiatric Association, 1994, Diagnostic and Statistical Manual of Mental Disorders, 4th ed. Washington, DC, APA.

Becker, D., Grilo, C., Edell, W., McGlashan, T., 2002, Diagnostic efficiency of borderline personality disorder criteria in hospitalized adolescents : Comparison with hospitalized adults, American Journal of Psychiatry, 159, 2042-2046.

Bernstein, D., Cohen, P., Velez, C., Schwab-Stone, M., Siever, L., Shinsato, L., 1993, Prevalence and stability of the DSM-III-R personality disorders in a community-based survey of adolescents, American Journal of Psychiatry, 150, 1237-1243.

Besser, A., Blatt, S. J., 2007, Identity consolidation and internalizing and externalizing problem behaviors in early adolescence, Psychoanalytic Psychology, 24, 1, 126-149.

BLos, P., 1967, The second individuation process of adolescence, Psychoanalytic Study of the Child, 22, 162-186.

Borenstein, R., 1992, The dependent personality: Development of social and clinical perspectives, Psychological Bulletin, 112, 3-23.

Burns, B. J., HoAgwood, MrazeK, P. J., 1999, Effective treatment for mental disorders in children and adolescents, Clinical Child and Family Psychology Review, 2, 199-254.

Chabrol, H., Montovany, A., Chouicha, K., Mullet, E., 2001, Frequency of borderline personality disorder in a sample of French high school students, Canadian Journal of Psychiatry, 46, 847-849.

Chabrol, H., LeichSenRing, F., 2006, Borderline personality organization and psychopathic traits in nonclinical adolescents: Relationships of identity diffusion, primitive defence mechanisms and reality testing with callousness and impulsivity traits, Bulletin of the Menninger Clinic, 70, $2,160-170$. 
Clarkin, J. F., 1996, Treatment of personality disorders, British Journal of Clinical Psychology, 33, 641-642.

Clarkin, J., Friedman, R., Hurt, S., Corn, R., Aronoff, M., 1986, Affective and character pathology of suicidal adolescent and young adult inpatients, Journal of Clinical Psychiatry, 45, 19-22.

Clarkin, J. F., Foelsch, P. A., Levy, K. N., Hull, J., Delaney, J., Kernberg, O. F., 2002, The development of a psychodynamic treatment for patients with borderline personality disorders : A preliminary study of behavioral change, Journal of Personality Disorders, 15, 6, 487-496.

Clarkin, J. F., Levy, K. N., Lenzenweger, M. F., Kernberg, O. F., 2004, The Personality Disorders Institute/Borderline Personality Disorder Research Foundation randomized control trial for borderline personality disorder: Rationale, methods, and patient characteristics, Journal of Personality Disorders, 18, 52-72.

Clarkin, J. F., Levy, K. N., LenZenweger, M. F., Kernberg, O. F., sous presse, Evaluating three treatments fro borderline personality disorder: A multiwave study, American Journal of Psychiatry.

Clarkin, J. F., Widiger, T., Frances, A., Hurt, S. W., Gilmore, M., 1983, Prototypic typology and the borderline personality disorder, Journal of Abnormal Psychology, 92, 263-275.

Clarkin, J. F., Yeomans, F. E., Kernberg, O. F., 1999, Psychotherapy for Borderline Personality, New York, Wiley and Sons.

Clarkin, J. F., Yeomans, F. E., Kernberg, O. F., 2006, Psychotherapy of Borderline Personality: Focusing on Object Relations, Washington, D.C., American Psychiatric Publishing Inc.

Cohen, P., Cohen, J., Kasen, S., Velez, C. N., Hartmark, C., Johnson, J., 1993, An epidemiological study of disorders in late childhood and adolescence. I. Age and gender-specific prevalence, Journal of Child Psychology and Psychiatry, 34, 851-867.

Coleman, J., 1993, Adolescence in a changing world, in Jackson, S. and Rodriguez-Tome, H., eds., Adolescence and its Social Worlds, Hove, UK, Erlbaum.

Diamond, D., Clarkin, J., Levine, H., Levy, K., Foelsch, P., Yeomans, F., 1999, Borderline Conditions and Attachment: A Preliminary Report, in Diamond, D., Blatt, S.J., eds., Attachment Research and Psychoanalysis, Psychoanalytic Inquiry, 19, 5, 831-884.

Diamond, D., Clarkin, J. F., Stovall-McClough, K. C., Levy, K. N., Foelsch, P. A., Levine, H., Yeomans, F. E., 2003, Patient-Therapist 
attachment: impact on the therapeutic process and outcome, in Mauricio Cortina, ed., Attachment Theory and the Psychoanalytic Process, Taylor and Francis.

ERICKSON, E. H., 1950, 1985, The eight ages of man, Childhood and Society, W. W. Norton, New York, 247-274.

ERICKSON, E. H., 1959, The theory of infantile sexuality, Childhood and Society, W. W. Norton, New York, 42-92.

Erickson, E. H., 1982, The Life Cycle Completed: A Review, W. W. Norton, New York.

Fergusson, D. M., Horwood, L. J., Ridder, E. M., Beautrais, A. L., 2005, Subthreshold depression in adolescence and mental health outcome in adulthood, Archives of General Psychiatry, 62, 1, 66-72.

Fleming, J., OfFord, D. R., 1990, Epidemiology of childhood depressive disorders: A critical review, Journal of the American Academy of Child and Adolescent Psychiatry, 29, 571-580.

Fonagy, P., Gergely, G., Jurist, E. L., Target, K., 2002, Affect Regulation, Mentalization, and the Development of the Self, Other Press LLC, New York.

Hurt, S., Clarkin, J., Munroe-Blum, H., Marziali, R., 1992, Borderline behavioral clusters and different treatment approaches, in Clarkin, J., Marziali, E., Munroe-Blum, H., eds., Borderline Personality Disorder: Clinical and Empirical Perspectives, Gilford Press, New York, 199-219.

Kernberg, O., 1976, Object Relations Theory and Clinical Psychoanalysis, Aronson, New York.

Kernberg, O., 1977, The structural diagnosis of borderline personality organization, in. Hartocollis, M.P., ed., Borderline Personality Disorders: The Concept, the Syndrome, the Patient, I.U.P., New York.

KERNBERG, O., 1978, The diagnosis of borderline conditions in adolescence, in Feinstein, S., Giovacchini, P., eds., Adolescent Psychiatry, 6, University of Chicago Press, 298-319.

Kernberg, P., 1994, Psychological interventions for the suicidal adolescent, American Journal of Psychotherapy, 48, 1, 52-63.

Kernberg, P. F., Koenisgberg, H., 1999, The Extensive Identity Diffusion: On a Particular Form of Identity Diffusion in Borderline Ppatients Extending the Limits of Treatability, Basic Books, New York.

Kernberg, P. F., Weiner, A. S., Bardenstein, K. K., 2000, Personality Disorders in Children and Adolescents, Basic Books, New York. 
Lenzenweger, M. F., Loranger, A. W., Korfine, L., NefF, C., 1997, Detecting personality disorders in a non-clinical population: An application of a 2stage case identification, Archives of General Psychiatry, 54, 4, 345-351.

Lewinsohn, P. M., Rohde, P., SeEley, J. R., 1998, Major depressive disorder in older adolescents: Prevalence, risk factors, and clinical implications, Clinical Psychology Review, 18, 7, 765-794.

Lonigan, C. J., Elbert, J. C., Johnson, S. B., 1998, Special issue on empirically supported psychosocial interventions for children, Journal of Child Psychology, 27, 138-145.

Marton, P., Korenblum, M., Kutcher, S., Stein, B., Kennedy, B., Pakes, J., 1989, Personality dysfunction in depressed adolescent, Canadian Journal of Psychiatry, 34, 810-813.

McGlashan, T. H., 1996, The Chestnut Lodge followup study: III. Long-term outcome of borderline personalities, Archives of General Psychiatry, 43, 20-30.

McManus, M., Lerner, H., Robbins, D., Barbour, C., 1984, Assessment of borderline symptomatology in hospitalized adolescents, Journal of the American Academy of Child Psychiatry, 23, 685-694.

Michael, K. D., Crowley, S. L., 2002, How effective are treatments for child and adolescent depression? A meta-analytic review, Clinical Psychology Review, 22, 247-269.

Miller, A., 1999, Dialectical Behavioral Therapy: A new treatment approach for suicidal adolescents, American Journal of Psychotherapy, 53, 413418.

Miller, A., Glinski, J., Woodberry, K. A., Mitchell, A. G., Indik, J., 2002, Family therapy and dialectical behavior therapy with adolescents : Part I : Proposing a clinical synthesis, American Journal of Psychotherapy, 56, 568-585.

Mufson, L., Myrna, M.W., Moreau, D., Garfinkel, R., 1999, Efficacy of interpersonal psychotherapy for depressed adolescents, Archives of General Psychiatry, 56, 573-579.

OrbaCH, I., 2007, From abandonment to symbiosis : A developmental reversal in suicidal adolescents, Psychoanalytic Psychology, 24, 1, 150-166.

Pinto, A., Grapentine, L., Francis, G., Picariello, C., 1996, Borderline personality disorder in adolescents: Affective a cognitive features, Journal of the American Academy of Child and Adolescent Psychiatry, 35, 1338-1343. 
RUTTER, J., 1986, The development of psychopathology of depression: Issues and perspectives, in Rutter, M., Izard, C.E., Read, P.B., eds., Depression in Young People, Guilford Press, New York, 3-30.

Selzer, M. A., Kernberg, P. F., Fibel, B., Cerbuliez, T., Mortati, S., 1987, The personality assessment interview: preliminary review, Psychiatry, $50,2,142-153$.

Skodol, A., Gunderson, J., Pfohl, B., Widiger, T. A., Livesley, W. J., Siever, L. J., 2002, The borderline diagnosis II : Biology, genetics, and clinical course, Biological Psychiatry, 51, 951-963.

Soloff, P. H., Lynch, K. G., Kelly, T. M., 2000, Psychopharmacology of borderline personality disorder, Psychiatric Clinical of North America, 23, 169-192.

Steinberg, L., 1996, Adolescence, 4th ed., McGraw Hill, New York.

Stone, M. H., 1993, Long-term outcome in personality disorders, British Journal of Psychiatry, 162, 299-313.

Torgersen, S., Kinglen, E., Cramer, V., 2001, The prevalence of personality disorders in a community sample, Achieves of General Psychiatry, 58, 590-596.

Weisz, J. R., Hawley, K. M., Jensen-Doss, A. L., 2004, Evidence update: Empirically tested psychotherapies for youth internalizing and externalizing problems and disorders, Child and Adolescent Psychiatric Clinics of North America, 13, 729-815.

Weisz, J. R., McCarty, C. A., Valeri, S. M., 2006, Effects of psychotherapy for depression in children and adolescents: A meta-analysis, Psychological Bulletin, 132, 1, 132-149.

Westen, D., 2006, Personality Pathology in Adolescence, Presented at the Unmasking Personality Disorders in Adolescents, New York.

Widiger, T. A., Weissman, M. M., 1991, Epidemiology of borderline personality disorder, Hospital Community Psychiatry, 42, 1015-1021.

WiKIPEDIA, From Wikipedia, the free encyclopedia. Retrieved November 2, 2006 from http"//en. wikipedia, org, wiki/Emo_(slang)

Woodberry, K., Miller, A., Glinski, J., Indik, J., Mitchell, A., 2002, Family therapy and dialectical behavior therapy with adolescents: Part II : A theoretical review, American Journal of Psychiatry, 56, 585-603.

Yeomans, F. E., Clarkin, J. F., KernberG, O. F., 2002, A Primer of TransferenceFocused Psychotherapy for the Borderline Patient, Aronson, New Jersey. 


\begin{abstract}
Treatment of Adolescents with Identity Diffusion : A Modification of Transference Focused Psychotherapy

This paper is an introduction to a treatment for adolescents with identity diffusion that has been modified from a therapy that has been found to be effective in the treatment of adults with significant identity diffusion, namely, Transference Focused Psychotherapy. The central role of differentiating normal Identity Crisis from Identity Diffusion in adolescents, essential to accurately identifying those adolescents appropriate for this treatment, is articulated. The primary modifications of TFP for adolescent treatment described involve changes in frequency and duration of some of the specific techniques (e.g. increased clarification; more work in the extra-transferential relationships before directly addressing the transference), tactics (e.g. inclusion of family during the assessment phase and treatment contract setting phases of treatment; inclusion of supportive interventions in the environment while maintaining the analytic stance in sessions), and strategies (e.g. the goal of removing blockages to the development of normal identity integration, not "forced maturation"). This treatment aims to improve adolescent's relationships with friends, parents, and teachers; help clarify life goals; acquire positive self-esteem; and be better prepared for entering love relationships.
\end{abstract}

\title{
RESUMEN
}

\section{Modificaciones a la psicoterapia focalizada en la transferencia (PFT) para el tratamiento de adolescentes con identidad difusa}

Este artículo presenta una versión modificada de la psicoterapia focalizada en la transferencia (PFT) aplicada en los adolescentes que presentan una identidad difusa. Este método ya ha demostrado su eficacia en los adultos con importantes problemas de difusión de la identidad. Señalamos la importancia de hacer la distinción entre la crisis de identidad normal en la adolescencia y la difusión de la identidad, a fin de localizar bien a los adolescentes que podrían beneficiarse con este tratamiento. Las principales modificaciones aportadas a la PFT para adaptarse a los adolescentes descritos implican diversos cambios: cambios en cuanto a la frecuencia y duración de ciertas técnicas particulares (por ejemplo, una clarificación aumentada; una mayor atención a las relaciones extra-transferenciales antes de abordar directamente la transferencia), los cambios en cuanto a las tácticas (por ejemplo, la inclusión de la familia en la etapa de la evaluación y en el 
contrato que establece las fases del tratamiento, la inclusión de intervenciones de apoyo en el medio al mismo tiempo que se mantiene la posición analítica en el curso de las sesiones), los cambios en cuanto a las estrategias (por ejemplo, intentar remover los bloqueos que perjudican el desarrollo normal de la integración de la identidad, en lugar de "forzar la maduración"). El tratamiento busca mejorar las relaciones del adolescente con sus amigos, padres y profesores; clarificar sus objetivos en la vida; adquirir una estima de sí mismo y estar mejor preparado para comprometerse en las relaciones amorosas.

\section{RESUMO}

\section{Modificações na psicoterapia focalizada na transferência (PFT) para o tratamento de adolescentes com uma identidade difusa}

Este artigo apresenta uma versão modificada da psicoterapia focalizada na transferência (PFT) aplicada em adolescentes que demostram ter uma identidade difusa. Este método já mostrou sua eficácia junto a adultos que sofrem de problemas graves de difusão da identidade. Chamamos a atenção para a importância de distinguir a crise identitária normal da adolescência da difusão da identidade, para detectar corretamente os adolescentes que poderiam se beneficiar deste tratamento. As principais modificações feitas na PFT para adaptá-la aos adolescentes descritos implicam várias mudanças: quanto à freqüência e à duração de algumas técnicas singulares (por exemplo, uma maior clarificação; uma maior atenção às relações extra-transferenciais antes de abordar diretamente a transferência), mudanças quanto às táticas (por exemplo, a inclusão da família na etapa da avaliação e no contrato que estabelece as fases do tratamento, a inclusão de intervenções de apoio no meio, mantendo a posição analítica durante as sessões), mudanças quanto às estratégias (por exemplo, tentar retirar bloqueios que atrapalham o desenvolvimento normal da integração da identidade, ao invés de "forçar a amadurecimento"). O tratamento visa melhorar as relações dos adolescentes com seus amigos, pais e professores; a esclarecer seus objetivos na vida; a adquirir auto-estima e estar melhor preparado para investir nas relações amorosas. 